\title{
New insight into the features of Behçet's disease with gastrointestinal ulcer: a cross-sectional observational study
}

\author{
Jing-Fen Ye ${ }^{1}$, Cheng-Cheng Hou' ${ }^{1}$, Hua-Fang Bao ${ }^{2}$ and Jian-Long Guan ${ }^{{ }^{*}}$ (i)
}

\begin{abstract}
Background: Behçet's disease (BD) can involve any site of the alimentary canal. There has been research concerning intestinal BD. Nevertheless, the entire digestive tract not yet been studied extensively. Therefore, the purpose of study was to describe the prevalence, location, clinical features and possible risk factors of $\mathrm{BD}$ with gastrointestinal tract ulcer.

Methods: This was a cross-sectional observational study that included 1232 consecutive BD patients who routinely underwent endoscopy upon their wishes. The clinical symptoms, endoscopic findings, and histologic features of BD with gastrointestinal ulcer and negative Helicobacter pylori ( $\mathrm{Hp})$ were identified.

Result: We found that 22.16\% (273/1232) BD patients had ulcers of the alimentary tract. At presentation, 61.54\% (168/273) patients were asymptomatic. Isolated gastroduodenal involvement is an extremely usual event. The second was the pairwise combination between bowel segments, and 24 cases involved three segments at the same time. One patient suffered from total gastrointestinal tract involvement. Inflammation was the most common histopathologic feature $77.60 \%(142 / 183)$. The $273 \mathrm{BD}$ patients with gastrointestinal ulcer were at greater risk of having archenteric symptoms (OR 0.070, $P<0.001)$, fever (OR 0.115, $P=0.047)$, high CRP (OR 0.994, $P=0.027)$ and BDCAF level (OR $0.590, P=0.010)$. Uveitis correlates negatively with gastrointestinal involvement in $B D$ patients $(O R 3.738, P=0.011)$.

Conclusions: BD could affect the upper gastrointestinal tract independently. Endoscopy should be conducted in all patients in whom a diagnosis of BD is entertained, especially in patients with higher CRP, disease activity and fever. While, BD patients with uveitis correlates negatively with gastrointestinal involvement.
\end{abstract}

Keywords: Behçet's disease, Gastrointestinal ulcer, Clinical characteristics, Risk factors

\section{Introduction}

Behçet's disease (BD) is a systemic vasculitis that can involve various organs at temporally distinct phases. Digestive tract ulcer, a common manifestation of BD, may develop about $4.5-6$ years after the onset of oral ulcerations [1]. Despite the fact that the ileocecal

\footnotetext{
*Correspondence: jianlong_guan@126.com

${ }^{1}$ Department of Immunology and Rheumatology Division, Fudan

University Huadong Hospital, \#221 Yan'an West Road, Shanghai 200040, People's Republic of China

Full list of author information is available at the end of the article
}

region is the part most often involved, involvement of the upper gastrointestinal tract and small intestine have also been reported [2-5]. To date, much research has been conducted to investigate the involvement of the lower gastrointestinal tract; however, the involvement of the entire gastrointestinal in BD has not been studied sufficiently. Clinical presentation varies, with the most common symptom of intestinal BD being abdominal pain with or without diarrhea. About $30 \%$ of intestinal BD patients present with emergency conditions such as hemorrhage or perforation [6]. The clinical manifestations of upper gastrointestinal tract original author(s) and the source, provide a link to the Creative Commons licence, and indicate if changes were made. The images or other third party material in this article are included in the article's Creative Commons licence, unless indicated otherwise in a credit line to the material. If material is not included in the article's Creative Commons licence and your intended use is not permitted by statutory regulation or exceeds the permitted use, you will need to obtain permission directly from the copyright holder. To view a copy of this licence, visit http://creativecommons.org/licenses/by/4.0/. The Creative Commons Public Domain Dedication waiver (http://creativeco mmons.org/publicdomain/zero/1.0/) applies to the data made available in this article, unless otherwise stated in a credit line to the data. 
involvement may present with odynophagia and substernal pain. A study showed that the prevalence of esophageal involvement in BD is very uncommon and that patients with such involvement face few complications [7]. The incidence of gastrointestinal tract involvement in $\mathrm{BD}$ is inconsistently reported, possibly because of population region or research criteria [6]. Another reason is that gastrointestinal ulcers frequently appear in asymptomatic patients. In a previous study, colonoscopy was performed in 401 consecutive BD subjects, 88 with intestinal lesions. Among the subjects with ulcers, $62.86 \%$ did not have any abdominal discomfort [8]. In routine practice, endoscopy is performed in BD patients merely with symptoms. Therefore, we suspect that the real frequency of gastrointestinal BD might be higher. The present study was designed to determine the prevalence, location, clinical features of digestive tract ulcers; we determined risk factors that predicted gastrointestinal involvement in consecutive BD patients undergoing endoscopy, irrespective of gastrointestinal symptoms.

\section{Methods and patients}

Data from the medical records of $1232 \mathrm{BD}$ patients who underwent endoscopy between October 2013 and April 2020 were collected from Huadong Hospital affiliated to Fudan University. Of 1232 subjects, 273 were finally enrolled into the gastrointestinal BD group after meeting the following criteria: (1) Enrolled patients met the international Criteria for Behçet Disease (ICBD) published in 2013 [9]; (2) In all cases, inclusion in the study required that the existence of gastrointestinal ulcer be confirmed endoscopically or surgically; (3) Subjects with any other gastrointestinal diseases such as intestinal tuberculosis, nonspecific colitis, intestinal cancer or reflux esophagitis were excluded; (4) Patients were also excluded if they had any history of taking Non-Steroidal Anti-Inflammatory Drugs (including low doses of aspirin) within 30 days before endoscopy and there is peptic ulcer under endoscopy. (5) Those who met BD and had upper gastrointestinal ulcers were excluded if they were positive for Hp by rapid urease test under Endoscopy (Fig. 1).

We reviewed the medical records of 1232 subjects, recording demographics, clinical manifestations and laboratory findings including ESR, CRP and $\mathrm{Hb}$. Clinical

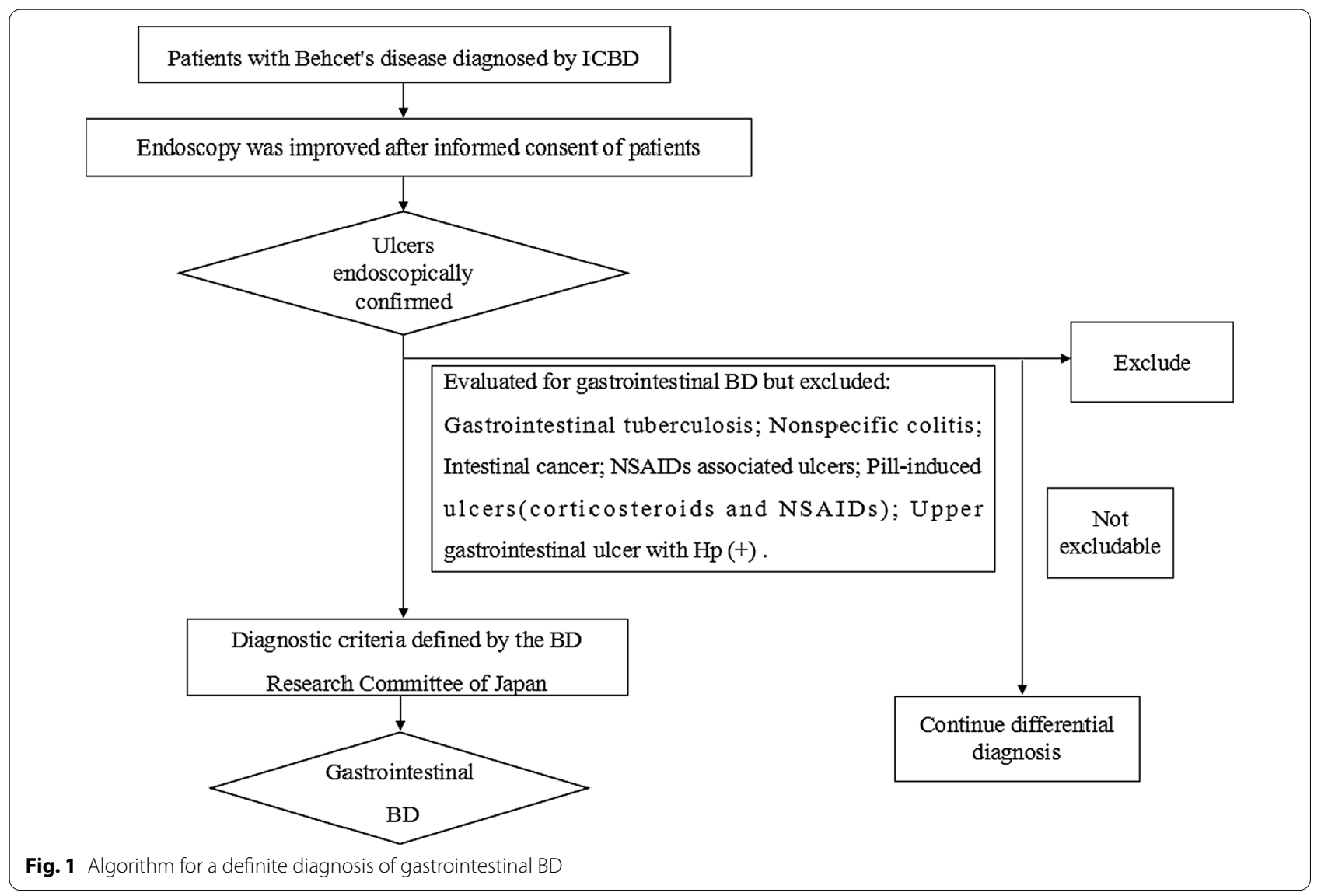


manifestations included gastrointestinal symptoms and various symptoms: fever, oral ulcers, genital ulcers, skin lesions, ophthalmitis, neurologic lesion, Myelodysplastic syndrome (MDS), venous thrombosis, valvulopathy and aneurysm. Disease locations and histopathology were also recorded in $273 \mathrm{BD}$ patients with gastrointestinal ulcer. This study protocol was approved by the ethics committee of the hospital.

\section{Statistical analysis}

Continuous variables were expressed as means with ranges. Discrete data were expressed as numbers or percentages or both. Demographics, clinical symptoms, and subjects with and without gastrointestinal ulcer in BD patients were compared. For comparative analyses, the Mann-Whitney U test and Student's t test were used for continuous variables, while the chi-squared test was used for categorical variables, where appropriate. A logistic regression model for prediction of gastrointestinal ulcer was computed. All statistical analyses were performed using SPSS 17.0. $\mathrm{P}<0.05$ was considered statistically significant.

\section{Results}

\section{The macroscopic and microscopic characteristics} of gastrointestinal ulcers in BD patients (Table 1; Fig. 1) Of 1232 BD patients, 22.16\% (273/1232) had gastrointestinal ulcers. An almost equal distribution between females and males was found in the group of BD patients with gastrointestinal ulcer. The mean age was $39.33 \pm$ 14.62 years. Gastrointestinal symptoms were noted in only $38.46 \%(105 / 273)$ in BD patients who had gastrointestinal ulcers confirmed by endoscopy or surgery. Of these, abdominal pain was the most common symptom $27.12 \%$ (74/273), followed by hematochezia $14.29 \%$ (39/273), diarrhea $12.09 \%$ (33/273), retrosternal pain $2.93 \%$ (8/273), dysphagia $2.56 \%$ (7/273), and nausea $0.37 \%(1 / 273)$. The diameter of ulcer ranged from needle-tip size to giant ulcer. Ulcers larger than or equal to $2 \mathrm{~cm}$ were recorded in $18(22.22 \%)$ patients. Round/oval shape was the most common 99 (76.74\%). Biopsy specimens were obtained from 183 patients. Inflammatory cell infiltration, including neutrophils, lymphocytes cells was the most common histopathologic feature 142 (77.60\%), followed by granuloma 39 (21.31\%). Vasculitis was found in only two patients (Table 1; Fig. 2a, b). The numbers of patients with BD had gastrointestinal ulcers isolated to the upper gastrointestinal tract, small bowel, ileocecal region, ascending colon, transverse colon, descending colon, sigmoid colon and rectum were $84,4,106,2,3,1$, 2 and 8 , respectively. The numbers of intestinal segments involved in the remaining patients were more than or equal to 2. Of these, 24 patients had three or more intestinal segments involved. One patient suffered from total gastrointestinal tract involvement (Fig. 3).

\section{Comparison of the demographic and clinical manifestations between BD patients with isolated upper gastrointestinal ulcers and isolated ileocecal involvement}

We identified $84 \mathrm{BD}$ patients with isolated upper gastrointestinal tract ulcer. Compared to controls with isolated ileocecal ulcers, these patients were younger at BD symptom onset $(P=0.008)$. An equal distribution between male and female was found, and no difference was noted between the two groups in terms of duration. The most frequent extraintestinal systemic manifestation of both groups was oral ulcer. Neurologic lesions were more frequent in BD patients with isolated ileocecal ulcers $(P$ $=0.015$ ), while skin lesions were more common in $\mathrm{BD}$ patients with isolated upper gastrointestinal tract ulcers $(P=0.035)$. The rates of complications including perforation $(P=0.004)$, obstruction $(P=0.015)$ and abdominal surgery at diagnosis were higher in BD patients with isolated ileocecal ulcers. There was no significant difference between the groups in terms of endoscopic pathological results, or levels of ESR, leukocytes, neutrophils, eosinophils, basophils, lymphocytes, monocytes, platelets, serum ferritin, and CRP (Table 2).

Table 1 The characteristics of BD patients with gastrointestinal ulcer

\begin{tabular}{ll}
\hline Characteristics $(\mathbf{n}=\mathbf{2 7 3}$ ) & \\
\hline Age (years) & $39.33 \pm 14.62$ \\
Gender (male/female) & $122 / 151$ \\
Gastrointestinal symptom n (\%) & $105(38.46)$ \\
Abdominal pain n (\%) & $74(27.12)$ \\
Diarrhea n (\%) & $33(12.09)$ \\
Hematochezia n (\%) & $39(14.29)$ \\
Nausea $\mathrm{n}(\%)$ & $1(0.37)$ \\
Retrosternal pain $\mathrm{n}(\%)$ & $8(2.93)$ \\
Swallow pain $\mathrm{n}(\%)$ & $7(2.56)$ \\
No gastrointestinal symptom n (\%) & $168(61.54)$ \\
Morphology of ulcer & \\
Approximate circle $\mathrm{n}(\%)$ & $99(76.74)$ \\
Irregular shape $\mathrm{n}(\%)$ & $19(14.73)$ \\
Annular ulcer $\mathrm{n}(\%)$ & $3(2.33)$ \\
Longitudinal ulcer $\mathrm{n}(\%)$ & $8(6.20)$ \\
Ulcer diameter $>2$ cm n (\%) & $18(22.22)$ \\
Pathological findings & \\
Vasculitis $\mathrm{n}(\%)$ & $2(1.09)$ \\
Granuloma $\mathrm{n}(\%)$ & $39(21.31)$ \\
Inflammatory infiltration $\mathrm{n}(\%)$ & $142(77.60)$ \\
\hline
\end{tabular}

Values are presented as number (\%) or mean \pm standard deviation 


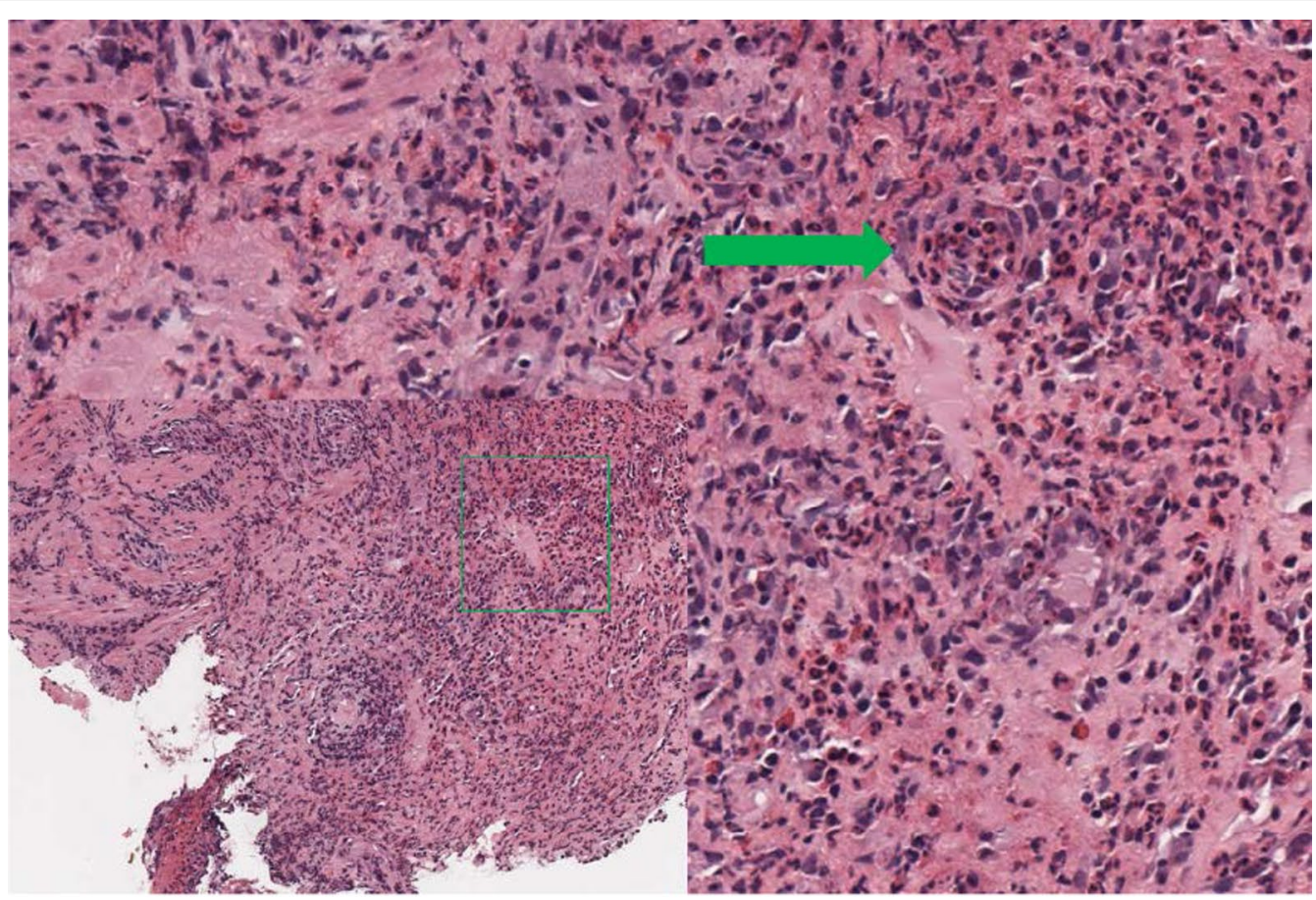

a

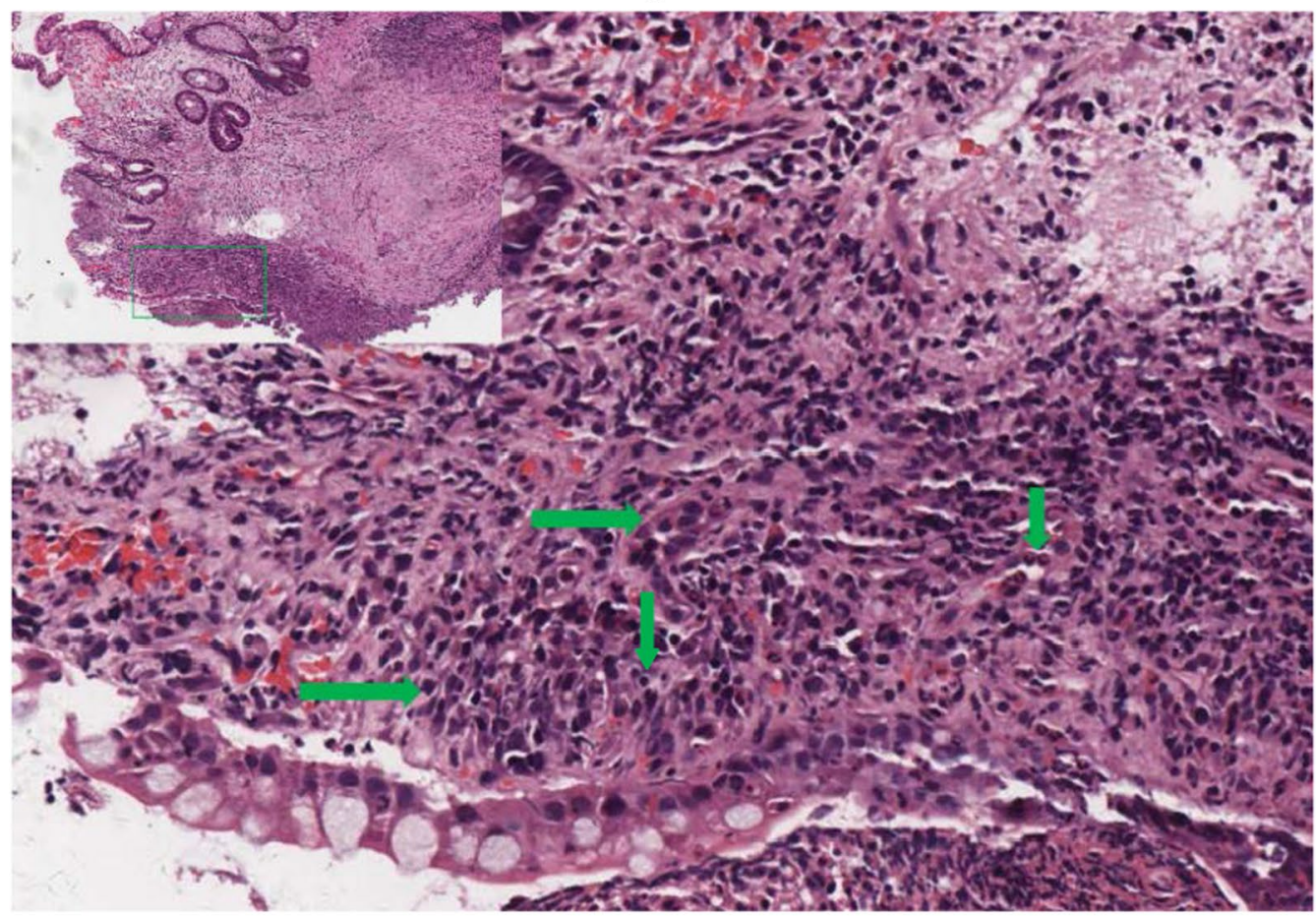

b

Fig. 2 a A large number of neutrophils infiltrated into the small blood vessels in the lamina propria of esophageal mucosa, and the small blood vessels were inflamed. $\mathbf{b}$ Histopathologic findings in colonoscopic biopsy specimens demonstrating plasma cell infiltration, interstitial small vessel swelling, lymphocyte infiltration and neutrophil infiltration (arrow from left to right) 


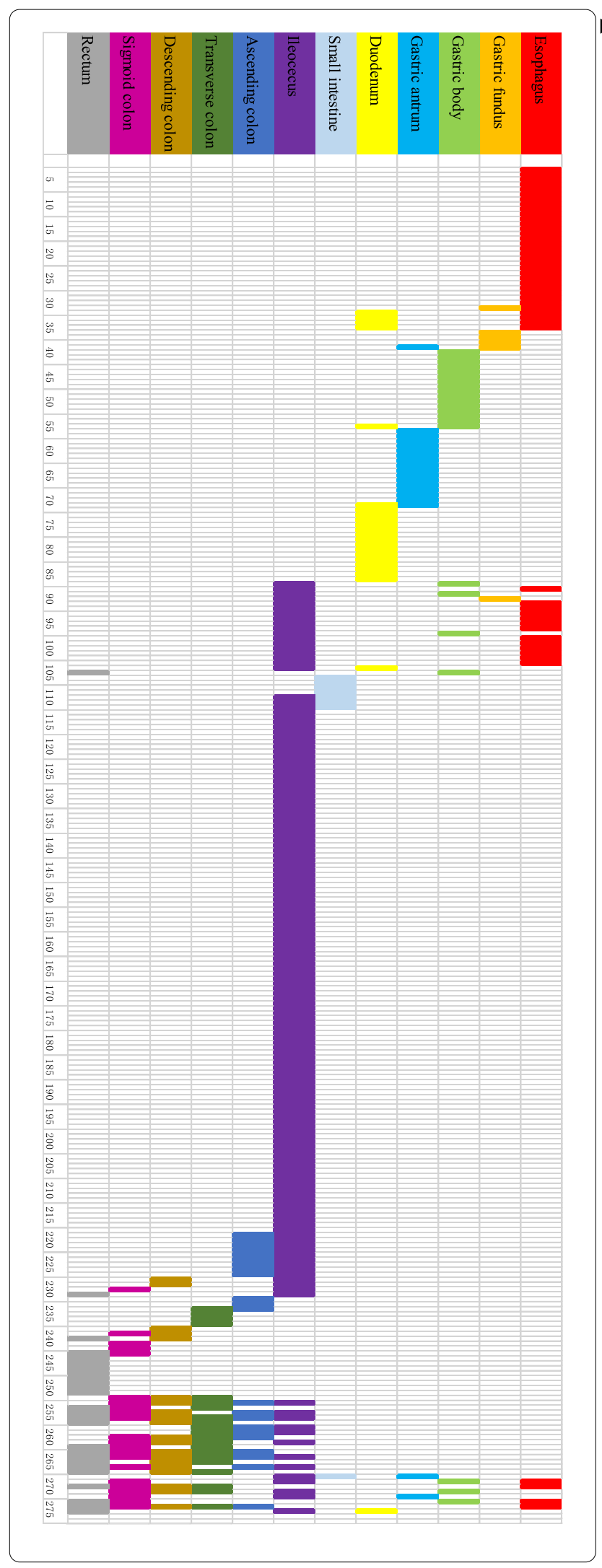

Fig. 3 Distribution of gastrointestinal ulcer in 273 patients with $B D$. Endoscopy showed that gastrointestinal ulcer in patients with $B D$ may occur in any part of the alimentary tract from the esophagus to the rectum. The main foci of the gastrointestinal ulcer in $\mathrm{BD}$ patients are typically the isolated terminal ileum and colon. The numbers of $\mathrm{BD}$ patients with gastrointestinal ulcers isolated to ileocecal region, ascending colon, transverse colon, descending colon, sigmoid colon and rectum were 106, 2, 3, 1,2 and 8, respectively. However, isolated upper gastrointestinal tract involvement, including the esophagus, gastric body, gastric body, gastric antrum, and duodenum, lesions are also detected as well, with a relatively high frequency $30.76 \%$ (84/273). 24 patients with multiple segment involvement

\section{Predictors associated with gastrointestinal ulcer in patients with Bechet's disease}

In a multivariate logistic regression model, gastrointestinal symptoms (OR 0.070, $P<0.001$ ), fever (OR $0.115, P$ $=0.047)$, and high levels of CRP (OR 0.994, $P=0.027)$ and BDCAF level (OR 0.590, $P=0.010$ ) were independent predictors for the presence of gastrointestinal ulcers. By contrast, Uveitis correlates negatively with gastrointestinal involvement in $\mathrm{BD}$ patients (OR 3.738, $P=0.011$ ) (Table 3).

\section{Discussion}

Behçet's disease (BD) is a chronic multisystem complex disorder. It has been classified in multiple phenotypes according to organ involvement (mucocutaneous, ophthalmic, cardiac, vascular, neurological, hematological, and gastrointestinal) through cluster and association studies $[10,11]$. Despite the fact that gastrointestinal ulcer is not part of the classification criteria for this disease, its incidence is not low and can easily lead to severe morbidity and mortality. BD is usually designated "entero-BD" if an ulcer in the gastrointestinal tract is identified, and is always classified as an independent phenotype [12]. To date, much research has been conducted to determine the involvement of the intestinal BD. Nevertheless, the full length of digestive tract in BD has not yet been studied extensively. In the present study, we investigated the clinical features and risk factors of $\mathrm{BD}$ with gastrointestinal ulcers.

We identified 273 (22.16\%) patients with gastrointestinal ulcers, a higher proportion than reported previously [13]. We speculate that the differences are due to the fact that previous studies mostly involved endoscopy in symptomatic BD patients [14], in addition to being based on dissimilar subject baseline characteristics such as race, geography, and differences in diagnostic criteria [6]. Consistent with other studies [4, 11], abdominal pain, hematochezia, diarrhea, retrosternal pain, and nausea can occur in BD patients with gastrointestinal ulcer. 
Table 2 Comparison between BD patients with isolated upper gastrointestinal ulcer and isolated ileocecus

\begin{tabular}{|c|c|c|c|}
\hline Characteristics & $\begin{array}{l}\text { BD patients with isolated upper } \\
\text { gastrointestinal ulcer }\end{array}$ & $\begin{array}{l}\text { BD patients with isolated ileocecus } \\
\text { ulcer }\end{array}$ & $P$ \\
\hline Number of patients & $\mathrm{n}=84$ & $n=106$ & \\
\hline \multicolumn{4}{|l|}{ Demographics } \\
\hline Gender (M/F) & $55 / 29$ & $56 / 50$ & 0.054 \\
\hline Age at onset (years, mean \pm SD) & $32.99 \pm 14.36$ & $27.33 \pm 14.63$ & 0.008 \\
\hline Duration (months, mean \pm SD) & $108.70 \pm 100.32$ & $114.72 \pm 122.27$ & 0.716 \\
\hline \multicolumn{4}{|l|}{ Clinical manifestations } \\
\hline \multicolumn{4}{|l|}{ Extraintestinal manifestations } \\
\hline Oral ulcers n (\%) & $84(100)$ & $106(100)$ & \\
\hline Genital ulcers n (\%) & $57(67.86)$ & $71(66.98)$ & 0.512 \\
\hline Skin lesions n (\%) & $42(50)$ & $38(35.85)$ & 0.035 \\
\hline Ocular lesions n (\%) & $5(5.95)$ & $6(5.66)$ & 0.343 \\
\hline Neurologic lesions n (\%) & $0(0)$ & $7(6.60)$ & 0.015 \\
\hline Cardiac valvular lesions n (\%) & $1(1.19)$ & $0(0)$ & 0.442 \\
\hline Venous thrombosis n (\%) & $3(3.57)$ & $2(1.89)$ & 0.391 \\
\hline Aneurysms n (\%) & $3(3.57)$ & $1(0.94)$ & 0.229 \\
\hline \multicolumn{4}{|l|}{ Gastrointestinal complications } \\
\hline Hemorrhage n (\%) & $7(8.33)$ & $15(14.15)$ & 0.155 \\
\hline Perforation n (\%) & $0(0)$ & $9(8.49)$ & 0.004 \\
\hline Obstruction n (\%) & $0(0)$ & $7(6.60)$ & 0.015 \\
\hline History of surgery n (\%) & $1(1.19)$ & $11(10.38)$ & 0.008 \\
\hline \multicolumn{4}{|l|}{ Assistant examination } \\
\hline $\mathrm{ESR}(\mathrm{mm} / \mathrm{h})$ & $18(7.25 \sim 28.75)$ & $16(7.50 \sim 40.00)$ & 0.580 \\
\hline CRP (mg/L) & $8.55(3.19 \sim 10.00)$ & $10(3.68 \sim 19.65)$ & 0.167 \\
\hline Leukocyte & $6.06 \pm 2.39$ & $7.55 \pm 3.18$ & 0.084 \\
\hline Neutrophil & $57.54 \pm 15.38$ & $63.11 \pm 14.33$ & 0.463 \\
\hline Eosinophil & $2.36 \pm 2.16$ & $2.53 \pm 1.48$ & 0.220 \\
\hline Basophil & $0.475 \pm 0.37$ & $0.47 \pm 0.39$ & 0.401 \\
\hline Lymphocyte & $32.03 \pm 12.62$ & $26.43 \pm 12.53$ & 0.851 \\
\hline Monocyte & $7.59 \pm 3.03$ & $8.35 \pm 3.49$ & 0.522 \\
\hline Platelet & $231.91 \pm 72.00$ & $247.07 \pm 89.09$ & 0.277 \\
\hline Serum ferritin & $185.31 \pm 42.49$ & $185.40 \pm 44.17$ & 0.985 \\
\hline BDCAF & $3.28 \pm 0.924$ & $3.22 \pm 1.146$ & 0.100 \\
\hline Pathological findings & & & 0.055 \\
\hline Vasculitis n (\%) & $0(0)$ & $2(2.67)$ & \\
\hline Granuloma n (\%) & $9(15.25)$ & $21(28.00)$ & \\
\hline Inflammatory infiltration n (\%) & $50(84.75)$ & $52(69.33)$ & \\
\hline
\end{tabular}

$B D$ Behçet's disease, $S D$ standard deviation

$\mathbf{P}$ values with statistical significance are shown in bold in the table

However, the presence of gastrointestinal symptoms does not always accurately indicate the presence of gastrointestinal ulcer in BD patients. Similar phenomena do not occur in the Chinese population merely $[15,16]$. Yi et al. reported that only half of the BD patients with upper gastrointestinal symptoms did actually have esophageal involvement [6]. Therefore, the ability of gastrointestinal symptoms to predict the presence of gastrointestinal ulcers is limited. We further analyzed the clinical risk factors of $\mathrm{BD}$ patients with gastrointestinal ulcer and found that the higher CRP and fever suggested the presence of gastrointestinal lesions. This is in accordance with results of a previous study [12]. Compared with patients without GI involvement, fewer ocular lesions, lower levels of albumin, erythrocyte counts and hemoglobin, and higher levels of CRP and ESR were found in the intestinal BD group [12]. These results may aid the establishment of guidelines for gastrointestinal examination among $\mathrm{BD}$ 
Table 3 Factors for prediction of gastrointestinal ulcer in BD patients

\begin{tabular}{|c|c|c|c|c|}
\hline Variable & $\begin{array}{l}\text { BD patients without } \\
\text { gastrointestinal ulcer }\end{array}$ & $\begin{array}{l}\text { BD patients with } \\
\text { gatrointestinal ulcer }\end{array}$ & $\begin{array}{l}\text { Univariate analysis, } \\
P \text { value }\end{array}$ & $\begin{array}{l}\text { Multivariate analysis, } \\
P \text { value }\end{array}$ \\
\hline $\operatorname{Sex}(F / M)$ & $477: 482$ & $122: 151$ & 0.080 & \\
\hline Age at diagnosis & $38.91 \pm 13.53$ & $39.33 \pm 14.62$ & 0.660 & \\
\hline Smoking history & $87: 872$ & $28: 245$ & 0.312 & \\
\hline Archenteric symptoms & $913: 46$ & 168:105 & $<0.001$ & $\begin{array}{l}<0.001 \\
\text { OR } 0.070\end{array}$ \\
\hline Oral ulcer n (\%) & 949 (98.96) & $269(98.53)$ & 0.377 & \\
\hline Genital ulcer (\%) & $659(68.72)$ & $179(65.56)$ & 0.181 & \\
\hline Skin lesion (\%) & $471(49.11)$ & $117(42.85)$ & 0.039 & n.s \\
\hline Ocular (\%) & $164(17.10)$ & $16(5.86)$ & $<0.001$ & $\begin{array}{l}0.011 \\
\text { OR } 3.738\end{array}$ \\
\hline Hematological (MDS/+8) (\%) & $9(0.94)$ & $12(4.40)$ & $<0.001$ & n.s \\
\hline Cardiac valvular lesions (\%) & $25(2.61)$ & $2(0.73)$ & 0.041 & n.s \\
\hline Venous thrombosis (\%) & $24(2.50)$ & $9(3.30)$ & 0.297 & \\
\hline Aneurysms (\%) & $32(3.34)$ & $7(2.56)$ & 0.338 & \\
\hline Fever (\%) & $9(0.94)$ & $32(11.72)$ & $<0.001$ & $\begin{array}{l}0.047 \\
\text { OR } 0.115\end{array}$ \\
\hline $\operatorname{ESR}(\mathrm{mm} / \mathrm{h})$ & $12(6 \sim 25)$ & $19(8 \sim 40)$ & 0.001 & n.s \\
\hline CRP (mg/L) & $6.5(1.86 \sim 10)$ & $9.7(3.75 \sim 17.8)$ & $<0.001$ & $\begin{array}{l}0.027 \\
\text { OR } 0.994\end{array}$ \\
\hline Hemoglobin (g/L) & $132.66 \pm 17.44$ & $127.69 \pm 20.72$ & $<0.001$ & n.s \\
\hline Leukocyte & $9.47 \pm 3.99$ & $7.15 \pm 3.20$ & 0.280 & \\
\hline Neutrophil & $59.05 \pm 12.15$ & $61.31 \pm 14.91$ & 0.006 & n.s \\
\hline Eosinophil & $2.02 \pm 1.72$ & $1.73 \pm 1.72$ & 0.708 & \\
\hline Basophil & $0.51 \pm 0.46$ & $0.61 \pm 0.47$ & 0.688 & \\
\hline Lymphocyte & $30.24 \pm 11.15$ & $30.18 \pm 12.85$ & 0.715 & \\
\hline Monocyte & $7.93 \pm 2.88$ & $8.20 \pm 3.43$ & 0.056 & \\
\hline Serum ferritin & $144.67 \pm 135.41$ & $306.94 \pm 198.77$ & 0.002 & n.s \\
\hline Platelet & $240.81 \pm 82.84$ & $240.81 \pm 158.00$ & 0.995 & \\
\hline BDCAF & $2.86 \pm 0.765$ & $3.20 \pm 1.07$ & $<0.001$ & $\begin{array}{l}0.010 \\
\text { OR } 0.590\end{array}$ \\
\hline
\end{tabular}

MDS myelodysplastic syndrome

$\mathbf{P}$ values with statistical significance are shown in bold in the table

patients. In addition, a study of 43 patients with BD from Japan, Haruko et al. reported that BD with gastrointestinal ulceration had a lower frequency of eye involvement and higher incidence of arthritis and vascular involvement than did BD without gastrointestinal lesions [17]. We also found that BD patients with uveitis correlates negatively with gastrointestinal involvement. Uveitis may be a protective factor for gastrointestinal ulcer in patients with BD. These findings may not reveal any causal relationships. The association of clinical features revealed in this study does not imply a causal relationship. However, it may hide the conclusion that uveitis and gastrointestinal involvement may have different pathogeneses. This requires us to further explore the various etiopathogenesis mechanism of $\mathrm{BD}$ between different clinical phenotypes.
Numerous clinical studies have described the macroscopic manifestations of gastrointestinal ulcers in BD patients. A few (number of ulcers $<5$ ), round shape, and discrete border are general characteristics of gastrointestinal ulcer with BD $[18,19]$, which is consistent with our findings. In addition to, we found that focal distribution is another macroscopic feature of gastrointestinal ulcers in BD $[18,20]$. Despite the fact that ulcers can be seen at any portion of the gastrointestinal tract, diffuse and continuous colonic involvement is rare [21]. Upper gastrointestinal tract ulcers in BD have been documented mostly in anecdotal studies report [3-5]. In this study we found that upper gastrointestinal tract involvement may occur either isolated $6.82 \%(84 / 1232)$ or together with other digestive tube locations $1.62 \%$ (20/1232). Compared with the typical isolated ileocecal ulcerations, there were no significant differences in terms of demography, parenteral 
manifestations, pathological biopsy, or levels of erythrocyte sedimentation rate and C-reactive protein. While, the rates of perforation, obstruction and history of surgery were less common in isolated upper gastrointestinal tract than in the isolated ileocecal group, as reported previously [7]. The exact proportion of small-bowel involvement in BD has not been well determined. We found small intestinal ulcer can exist alone or with ileocecus, which is consist with the past study [22]. Therefore, evaluation of the small bowel by capsule endoscopy is also necessary in all patients BD.

$\mathrm{BD}$ is considered as a neutrophilic vasculitis and the role of neutrophils in BD pathogenesis has been repeatedly suggested in recent years. Elevated IL-17 causes significant neutrophil and lymphocyte influx in neutrophil-mediated inflammatory responses [23]. The retained fecal products result in the characteristic chronic granulomatous inflammation and adaptive immune responses. Impaired digestion of bacteria and fungi by neutrophils can result in similar pathological and clinical pictures [24]. In our study, histopathologic examination of the biopsy specimens revealed signs of vasculitis in only two patients. The distribution of this pathological result may be related to superficial and narrow colonoscopic biopsies. The characteristic histopathologic lesions of intestinal BD usually require analysis of deep and wide tissue for diagnosis [25]. These pathological findings indicate local inflammatory activity around the gastrointestinal ulcer.

We analyzed a large number of patients in a singlecenter BD cohort. Stringent inclusion and exclusion criteria, standardized enrolment and completion by both patients and physicians minimized the limitations of an observational data analysis. Nevertheless, there remain some limitations. First, it was a a cross-sectional observational, hospital-based study with potential bias in its design. Second, this paper mainly analyzed macroscopic characteristics of the special phenotype of BD. Further research is necessary to determine the true pathogeneses of these phenomena.

\section{Conclusions}

Upper gastrointestinal tract ulcers in BD may present in the form of isolated segment involvement, with lower incidence of perforation, obstruction stenosis, and history of surgery than isolated ileocecal ulcer. Gastrointestinal symptoms are not necessary conditions for the presence of gastrointestinal ulcer in BD patients. Routine endoscopy is advisable irrespective clinical manifestations. If endoscopy is not available, BD patients who develop fever, digestive tract symptoms, and elevated CRP should be strongly recommended to avoid serious complications caused by delayed diagnosis and treatment.

\section{Acknowledgements}

The authors had nothing to disclose.

\section{Authors' contributions}

YJF wrote the paper. HCC designed the study. BHF performed the statistical analysis. GJL conceived of the study, and participated in its design and coordination and helped to draft the manuscript. All authors read and approved the final manuscript.

\section{Funding}

This work is supported by Clinical Science Innovation Program of Shanghai Shenkang Hospital Development Center (SHDC12017129) and by grants from Project of East China Hospital Affiliated to Fudan University (2019lc028).

Availability of data and materials

Please contact author for data requests.

\section{Declarations}

Ethics approval and consent to participate

This work was approved by the medical ethics committee of Huadong Hospital affiliated to Fudan University with the following reference numbers: 2016 K044 and 2018 K031.

\section{Consent for publication}

Not applicable in this section.

\section{Competing interests}

The authors declare that they have no competing interests.

\section{Author details}

'Department of Immunology and Rheumatology Division, Fudan University Huadong Hospital, \#221 Yan'an West Road, Shanghai 200040, People's Republic of China. ${ }^{2}$ Division of Immunology and Rheumatology, Shanghai Key Laboratory of Clinical Geriatric Medicine, Research Center on Aging and Medicine, Huadong Hospital, Fudan University, No. 221 West Yan'an Road, Shanghai 200040, People's Republic of China.

Received: 23 July 2021 Accepted: 30 September 2021

Published online: 21 October 2021

\section{References}

1. Lopalco G, Rigante D, Venerito V, et al. Update on the medical management of gastrointestinal Behçet's disease. Mediators Inflamm. 2017. https://doi.org/10.1155/2017/1460491.

2. Arimoto J, Endo H, Kato T, et al. Clinical value of capsule endoscopy for detecting small bowel lesions in patients with intestinal Behcet's disease. Dig Endosc. 2016;28(2):179-85.

3. Hatemi I, Hatemi G, Erzin YZ, et al. Double pylorus in a patient with Behcet's syndrome. Clin Exp Rheumatol. 2015;33(6 Suppl 94):138-40.

4. Jia N, Tang Y, Liu H, et al. Rare esophageal ulcers related to Behcet disease: a case report. Med (Baltim). 2017;96(44):e8469.

5. Sonoda A, Ogawa R, Mizukami K, et al. Marked improvement in gastric involvement in Behcet's disease with adalimumab treatment. Turk J Gastroenterol. 2017:28(5):405-7.

6. Hatemi I, Hatemi Celik AF. Gastrointestinal involvement in Behçet's disease. Rheum Dis Clin N Am. 2018;44(1):45-64.

7. Yi SW, Cheon JH, Kim JH, et al. The prevalence and clinical characteristics of esophageal involvement in patients with Behcet's disease: a single center experience in Korea. J Korean Med Sci. 2009;24(1):52-6.

8. Chen Y, Liu WJ, Zou J, et al. Intestinal pathological changes in Behcet's disease: a clinical retrospective study. Fudan Univ J Med Sci. 2017:44:493-7. (in Chinese).

9. The International Criteria for Behcet's Disease (ICBD). a collaborative study of 27 countries on the sensitivity and specificity of the new criteria. J Eur Acad Dermatol Venereol. 2014;28(3):338-47.

10. Shen Y, Ma HF, Luo D, et al. High incidence of gastrointestinal ulceration and cytogenetic aberration of trisomy 8 as typical features of 
behcet's disease associated with myelodysplastic syndrome: a series of 16 consecutive Chinese patients from the Shanghai Behcet's Disease Database and comparison with the literature. Biomed Res Int. 2018. https://doi.org/10.1155/2018/8535091.

11. Seyahi E. Phenotypes in Behcet's syndrome. Intern Emerg Med. 2019;14(5):677-89.

12. Zou J, Shen Y, Ji DN, et al. Endoscopic findings of gastrointestinal involvement in Chinese patients with Behcet's disease. World J Gastroenterol. 2014:20(45):17171-8.

13. Hatemi I, Hatemi G, Celik AF. Gastrointestinal involvement in Behcet disease. Rheum Dis Clin N Am. 2018;44(1):45-64

14. Zhang Z, He F, Shi Y. Behcet's disease seen in China: analysis of 334 cases. Rheumatol Int. 2013;33(3):645-8.

15. Houman MH, Ben Gl, Lamloum M, et al. Esophageal involvement in Behcet's disease. Yonsei Med J. 2002;43(4):457-60.

16. Cakmak SK, Cakmak A, Gul U, et al. Upper gastrointestinal abnormalities and Helicobacter pylori in Behcet's disease. Int J Dermatol. 2009:48(11):1174-6.

17. Ideguchi H, Suda A, Takeno M, et al. Gastrointestinal manifestations of Behcet's disease in Japan: a study of 43 patients. Rheumatol Int. 2014;34(6):851-6.

18. Kim DH, Cheon JH. Intestinal Behcet's disease: a true inflammatory bowel disease or merely an intestinal complication of systemic vasculitis? Yonsei Med J. 2016;57(1):22-32

19. Hatemi I, Esatoglu SN, Hatemi G, et al. Characteristics, treatment, and long-term outcome of gastrointestinal involvement in Behcet's syndrome: a strobe-compliant observational study from a dedicated multidisciplinary center. Med (Baltim). 2016;95(16):e3348.

20. Valenti S, Gallizzi R, De Vivo D, et al. Intestinal Behcet and Crohn's disease: two sides of the same coin. Pediatr Rheumatol Online J. 2017;15(1):33-40.

21. Tominaga K, Kamimura K, Takahashi K, et al. A case of Behcet's disease with various gastrointestinal findings. Clin J Gastroenterol. 2018;11(5):354-8.

22. Arimoto J, Endo H, Kato T, et al. Clinical value of capsule endoscopy for detecting small bowel lesions in patients with intestinal Behet's disease. Dig Endosc. 2016;28(2):179-85.

23. Leccese P, Alpsoy E. Behcet's disease: an overview of etiopathogenesis. Front Immunol. 2019;10:1067. https://doi.org/10.3389/fimmu.2019.01067.

24. Segal AW. The role of neutrophils in the pathogenesis of Crohn's disease. Eur J Clin Invest. 2018;48(Suppl 2):e12983. doi:https://doi.org/10.1111/eci. 12983.

25. Hakim S, Ramireddy S, Amin M, et al. Preoperative misdiagnosis of intestinal Behcet's syndrome as Crohn's disease based on superficial colonoscopic biopsies: case report and systematic review. Dig Dis Sci. 2018;63(12):3509-15.

\section{Publisher's Note}

Springer Nature remains neutral with regard to jurisdictional claims in published maps and institutional affiliations.
Ready to submit your research? Choose BMC and benefit from:

- fast, convenient online submission

- thorough peer review by experienced researchers in your field

- rapid publication on acceptance

- support for research data, including large and complex data types

- gold Open Access which fosters wider collaboration and increased citations

- maximum visibility for your research: over $100 \mathrm{M}$ website views per year

At BMC, research is always in progress.

Learn more biomedcentral.com/submissions 